PROCEEDINGS OF THE

AMERICAN MATHEMATICAL SOCIETY

Volume 133, Number 2, Pages 455-464

S 0002-9939(04)07552-5

Article electronically published on August 30, 2004

\title{
SELF-ADJOINTNESS OF THE PERTURBED WAVE OPERATOR ON $L^{2}\left(\mathbf{R}^{\mathbf{n}}\right), n \geq 2$
}

\author{
MOHAMMED HICHEM MORTAD
}

(Communicated by Joseph A. Ball)

\begin{abstract}
We give classes of unbounded real-valued $V$ for which $\square+V$ is self-adjoint on $\mathcal{D}(\square) \subset L^{2}\left(\mathbf{R}^{\mathbf{n}}\right), n \geq 2$, where $\square$ is the wave operator defined on $\mathbf{R}^{\mathbf{n}}$.
\end{abstract}

\section{INTRODUCTION}

There are many classes of $V$ for which $-\Delta+V$ is self-adjoint on $\mathcal{D}(-\Delta)$. In this paper we investigate the self-adjointness of $\square+V$ where $\square=\frac{\partial^{2}}{\partial t^{2}}-\frac{\partial^{2}}{\partial x_{2}^{2}}-\ldots-\frac{\partial^{2}}{\partial x_{n}^{2}}$ is the usual wave operator and $V$ is an unbounded real-valued function defined on $\mathbf{R}^{\mathbf{n}}$. By using Fourier transforms on $L^{2}\left(\mathbf{R}^{\mathbf{n}}\right)$ one can show that $\square$ is selfadjoint on $\mathcal{D}(\square)=\left\{f \in L^{2}\left(\mathbf{R}^{\mathbf{n}}\right): \square f \in L^{2}\left(\mathbf{R}^{\mathbf{n}}\right)\right\}$ (we understand that the partial derivative is taken in the distributional sense). In the sequel $\mathcal{D}(\square)$ will be denoted by $M^{n}$ where $n$ stands for the dimension. We assume definitions and notions about unbounded operators, Fourier transforms, $B M O$ and interpolation theory, and for references one may consult [2], 3] or 4]. We also recall without proof the KatoRellich perturbation theorem (for a proof see [3], Theorem X.12), but before that we have

Definition 1. Let $A$ and $B$ be densely defined linear operators on a Hilbert space $H$. Suppose that:

i) $D(A) \subset D(B)$;

ii) for some $a$ and $b$ in $\mathbf{R}$ and all $\varphi \in D(A)$,

$$
\|B \varphi\| \leq a\|A \varphi\|+b\|\varphi\| \text {. }
$$

Then $B$ is said to be $A$-bounded. The infimum of such an $a$ is called the relative bound of $B$ with respect to $A$.

Theorem 1 (Kato-Rellich theorem). Suppose that $A$ is self-adjoint, $B$ is symmetric, and $B$ is $A$-bounded with relative bound $a<1$. Then $A+B$ is self-adjoint on $D(A)$.

Remark 1. Throughout this paper, $\tilde{c}$ will denote any arbitrary constant that need not be the same each time.

Received by the editors July 8, 2003 and, in revised form, October 1, 2003.

2000 Mathematics Subject Classification. Primary 47B25, 47A55, 46B70; Secondary 35L05, $32 \mathrm{~A} 37,46 \mathrm{E} 35$. 


\section{2. $M^{2}$ AND THE SPACE $B M O$}

It is known that by a simple change of variables we can change $\frac{\partial^{2}}{\partial t^{2}}-\frac{\partial^{2}}{\partial x^{2}}$ into $\frac{\partial^{2}}{\partial \eta \partial \xi}$. So we will use $\frac{\partial^{2}}{\partial x \partial y}$ to mean $\square$. We will prove that $M^{2} \subset B M O\left(\mathbf{R}^{2}\right)$, but first we have

Lemma 1. Fix $y_{1} \in \mathbf{R}$, and put $f_{1}(x)=\varphi\left(x, y_{1}\right)$ where $\varphi \in M^{2}$. Then $f_{1} \in$ $B M O(\mathbf{R})$ with uniform $B M O$ bound. Also $y \mapsto g_{1}(y)=\varphi\left(x_{1}, y\right)$ is in $B M O(\mathbf{R})$.

Proof. We have to prove $\frac{1}{|I|} \int_{I}\left|f_{1}(x)-\overline{f_{1}}\right| d x \leq c$ where $c$ does not depend on $y_{1}$, and where $\overline{f_{1}}=\frac{1}{|I|} \int_{I} f_{1}(x) d x$. By putting $\rho=\frac{\partial^{2} \varphi}{\partial x \partial y}$ we have

$$
\left(f_{1}-f\right)^{\prime}(x)=\int_{y}^{y_{1}} \rho(x, z) d z \text { where } f(x)=\varphi(x, y)
$$

and where we have the freedom to choose the $y$ that is convenient to us. Then we have

$$
\left\|\left(f_{1}-f\right)^{\prime}\right\|_{2}^{2} \leq\left(y_{1}-y\right)\|\square \varphi\|_{2}^{2}<\infty \text { with the assumption } y<y_{1} \text {. }
$$

So $\left(f_{1}-f\right)^{\prime} \in L^{2}(\mathbf{R})$. Hence, $f_{1}-f$ is continuous and so there exists a $c \in I$ such that

$$
\left(f_{1}-f\right)(x)-\overline{f_{1}-f}=\left(f_{1}-f\right)(x)-\left(f_{1}-f\right)(c),
$$

and since $\left(f_{1}-f\right)^{\prime} \in L_{l o c}^{1}(\mathbf{R})$ we have

$$
\left(f_{1}-f\right)(x)-\left(f_{1}-f\right)(c)=\int_{c}^{x}\left(f_{1}-f\right)^{\prime}(t) d t .
$$

By taking $I=(a, b)$ and since both $c$ and $x$ are in $I$, one has

$$
\left|\left(f_{1}-f\right)(x)-\left(f_{1}-f\right)(c)\right| \leq(b-a)^{\frac{1}{2}}\left\|\left(f_{1}-f\right)^{\prime}\right\|_{2}=|I|^{\frac{1}{2}}\left\|\left(f_{1}-f\right)^{\prime}\right\|_{2} .
$$

Hence

$$
\frac{1}{|I|} \int_{I}\left|\left(f_{1}-f\right)(x)-\left(f_{1}-f\right)(c)\right| d x \leq|I|^{\frac{1}{2}}\left(y_{1}-y\right)^{\frac{1}{2}}\|\square \varphi\|_{2} .
$$

So in order to find a uniform bound for $\left\|f_{1}-f\right\|_{B M O}$ for this particular $I$, it suffices to take $y$ such that $\left(y_{1}-y\right)^{\frac{1}{2}} \leq \frac{1}{|I|^{\frac{1}{2}}}$, and in such a case we will have

$$
\frac{1}{|I|} \int_{I}\left|\left(f_{1}-f\right)(x)-\overline{\left(f_{1}-f\right)}\right| d x \leq\|\square \varphi\|_{2} \text {. }
$$

But our interest is in the function $f_{1}$ itself, not in $f_{1}-f$. We can find a $B M O$ bound for $f_{1}$ for this particular $I$ if we come to show that $f$ is $B M O$. We have

$$
\frac{1}{|I|} \int_{I}|f(x)-\bar{f}| d x \leq \frac{1}{|I|} \int_{I}|f(x)| d x+\frac{1}{|I|} \int_{I}\left(\frac{1}{|I|} \int_{I}|f(t)| d t\right) d x
$$

Then

$$
\frac{1}{|I|} \int_{I}|f(x)-\bar{f}| d x \leq \frac{2\|f\|_{2}}{|I|^{\frac{1}{2}}} .
$$

So far we have not used the fact that $\varphi \in L^{2}\left(\mathbf{R}^{2}\right)$ ! To have a uniform $B M O$ bound for $f$ we need for instance $\|f\|_{2} \leq c|I|^{\frac{1}{2}}(c>0$, to be determined). By 
applying a simple calculus argument, one can say that there exists a $y \in \mathbf{R},\left|y_{1}-y\right| \leq$ $\frac{1}{(b-a)}$ such that $\|f\|_{2} \leq 2\|\varphi\|_{2}|I|^{\frac{1}{2}}$. So

$$
\frac{1}{|I|} \int_{I}\left|f_{1}(x)-\overline{f_{1}}\right| d x \leq \tilde{c}\|\square \varphi\|_{2}+\tilde{c}\|\varphi\|_{2} .
$$

The same proof works for $g_{1}$.

Theorem 2. Let $\varphi \in M^{2}$. Then $\varphi \in B M O\left(\mathbf{R}^{2}\right)$ and

$$
\|\varphi\|_{B M O\left(\mathbf{R}^{2}\right)} \leq a\|\square \varphi\|_{2}+b\|\varphi\|_{2} .
$$

Proof. The proof follows from Lemma 1 and Fubini's Theorem.

Hence we have the following corollary whose proof is an immediate consequence of an interpolation theorem ([4], Theorem 6.8).

Corollary 1. Let $2 \leq p<\infty$. Then any $\varphi \in M^{2}$ will be in $L^{p}\left(\mathbf{R}^{2}\right)$, and we have

$$
\|\varphi\|_{p} \leq a\|\square \varphi\|_{2}+b\|\varphi\|_{2}
$$

where the constants $a$ and $b$ depend on $p$.

Remark 2. The case $p=\infty$ is excluded, as will be shown below.

Lemma 2. The constant a in Corollary 1 may be made as small as we want.

Proof. Take $\varphi_{\lambda}(x, y)=\varphi(\lambda x, \lambda y): \lambda>0$. We get

$$
\left\|\square \varphi_{\lambda}\right\|_{2}=\lambda\|\square \varphi\|_{2}, \quad\left\|\varphi_{\lambda}\right\|_{2}=\frac{1}{\lambda}\|\varphi\|_{2} \quad \text { and } \quad\left\|\varphi_{\lambda}\right\|_{p}=\frac{1}{\lambda^{\frac{2}{p}}}\|\varphi\|_{p} .
$$

Thus the estimate (1) applied to $\varphi_{\lambda}$ instead of $\varphi$ becomes

$$
\|\varphi\|_{p} \leq a \lambda^{\frac{2}{p}+1}\|\square \varphi\|_{2}+b \lambda^{\frac{2}{p}-1}\|\varphi\|_{2}, \quad \lambda>0, p \geq 2 .
$$

By taking $\lambda$ small enough, the constant in front of $\|\square \varphi\|_{2}$ will be arbitrarily small.

Theorem 3. Let $\square$ be the wave operator on $L^{2}\left(\mathbf{R}^{2}\right)$. Let $\epsilon>0$, and let $V: \mathbf{R}^{\mathbf{2}} \rightarrow \mathbf{R}$ be such that $V \in L^{2+\epsilon}\left(\mathbf{R}^{2}\right)$. Then $\square+V$ is self-adjoint on $\mathcal{D}(\square)$.

Proof. We have by Corollary 1 that

$$
\|\varphi\|_{p} \leq a\|\square \varphi\|_{2}+b\|\varphi\|_{2}, \text { for } 2 \leq p<\infty .
$$

Then by Hölder's inequality,

$$
\|V \varphi\|_{2} \leq\|V\|_{q}\|\varphi\|_{p} \leq a\|V\|_{q}\|\square \varphi\|_{2}+b\|V\|_{q}\|\varphi\|_{2},
$$

for $\frac{1}{2}=\frac{1}{p}+\frac{1}{q}$ or $q=\frac{2 p}{p-2}$. Since the constant in front of $\|\square \varphi\|_{2}$ can be made small enough so that we have $a\|V\|_{q}<1$, we conclude by the Kato-Rellich perturbation theorem that $\square+V$ is self-adjoint on $\mathcal{D}(\square)=M^{2}$.

Proposition 1. Let $V \in L^{2+\epsilon}\left(\mathbf{R}^{2}\right)+L^{\infty}\left(\mathbf{R}^{2}\right)$ be a real-valued function, $\epsilon>0$. Then $\square+V$ is self-adjoint on $\mathcal{D}(\square)$.

Proof. The same as for Theorem 3. 


\section{A Counterexample}

Proposition 2. Let $\varphi \in L^{2}\left(\mathbf{R}^{2}\right)$ be such that $\frac{\partial^{2} \varphi}{\partial x \partial y} \in L^{2}\left(\mathbf{R}^{\mathbf{2}}\right)$. Then $\varphi$ need not be essentially bounded on $\mathbf{R}^{\mathbf{2}}$.

Proof. We are going to build up the counterexample by using a linear interpolation. We define $(x, y) \mapsto \varphi(x, y)$ on $\mathbf{R} \times\left(y_{n}, y_{n+1}\right)$ by

$$
\varphi(x, y)=\frac{1}{y_{n+1}-y_{n}}\left[\left(y-y_{n}\right) f_{n+1}(x)-\left(y-y_{n+1}\right) f_{n}(x)\right] \text { where } f_{n}(x)=\varphi\left(x, y_{n}\right),
$$

and the $f_{n}$ and $y_{n}$ are to be defined below.

Hence on $\mathbf{R} \times\left(y_{1}, \infty\right)$ we have

$$
\|\varphi\|_{2}^{2}=\iint_{\mathbf{R} \times\left(y_{1}, \infty\right)}|\varphi(x, y)|^{2} d x d y=\sum_{1}^{\infty} \iint_{\mathbf{R} \times\left(y_{n}, y_{n+1}\right)}|\varphi(x, y)|^{2} d x d y .
$$

After some calculations the condition that makes $\varphi$ belong to $L^{2}$ is

$$
\sum_{1}^{\infty}\left(y_{n+1}-y_{n}\right)\left(\left\|f_{n}\right\|_{2}^{2}+\left\|f_{n+1}\right\|_{2}^{2}\right)<\infty
$$

We also have

$$
\begin{gathered}
\frac{\partial^{2} \varphi}{\partial x \partial y}=\frac{1}{y_{n+1}-y_{n}}\left(f_{n+1}^{\prime}-f_{n}^{\prime}\right) \in L^{2}\left(\mathbf{R} \times\left(y_{1}, \infty\right)\right) \text { if } \\
\sum_{1}^{\infty} \frac{1}{y_{n+1}-y_{n}}\left\|\psi_{n}^{\prime}\right\|_{2}^{2}<\infty \text { where } \psi_{n}(x)=f_{n+1}(x)-f_{n}(x) .
\end{gathered}
$$

Since $\psi_{n}(x)=f_{n+1}(x)-f_{n}(x)$, then $f_{n}(x)=-\sum_{n}^{\infty} \psi_{k}(x)$. We also want $f_{n} \notin$ $L^{\infty}(\mathbf{R})$ so that $\varphi \notin L^{\infty}\left(\mathbf{R}^{2}\right)$.

Take $\psi_{n}(x)= \begin{cases}\frac{e^{n}}{n} x+\frac{1}{n} & \text { if }-e^{-n} \leq x \leq 0, \\ -\frac{e^{n}}{n} x+\frac{1}{n} & \text { if } 0 \leq x \leq e^{-n} \\ 0 & \text { if }|x| \geq e^{-n}\end{cases}$

Hence $\left\|\psi_{n}^{\prime}\right\|_{2}^{2} \sim \frac{e^{n}}{n^{2}}$ and $\left\|\psi_{n}\right\|_{2}^{2} \sim \frac{e^{-n}}{n^{2}}$. We also have

$$
\left\|f_{n}\right\|_{2} \leq \sum_{k=n}^{\infty}\left\|\psi_{k}\right\|_{2}=a \sum_{k=n}^{\infty} \frac{e^{-\frac{n}{2}}}{n} \simeq \int_{n}^{\infty} \frac{e^{-\frac{x}{2}}}{x} d x \leq \frac{1}{n} \int_{n}^{\infty} e^{-\frac{x}{2}} d x \sim \frac{e^{-\frac{n}{2}}}{n} .
$$

Now if we choose $y_{n+1}-y_{n}=e^{n}$, then the series

$$
\sum_{1}^{\infty} \frac{1}{y_{n+1}-y_{n}}\left\|\psi_{n}^{\prime}\right\|_{2}^{2}=\sum_{1}^{\infty} \frac{1}{e^{n}} \times \frac{e^{n}}{n^{2}}=\sum_{1}^{\infty} \frac{1}{n^{2}}
$$

converges and so does

$$
\sum_{1}^{\infty}\left(y_{n+1}-y_{n}\right)\left(\left\|f_{n}\right\|_{2}^{2}+\left\|f_{n+1}\right\|_{2}^{2}\right) \leq \sum_{1}^{\infty} e^{n} \times\left[\frac{e^{-(n+1)}}{(n+1)^{2}}+\frac{e^{-n}}{n^{2}}\right] \sim \sum_{1}^{\infty} \frac{1}{n^{2}} .
$$

Now the $\varphi$ defined on $\mathbf{R} \times\left(y_{n}, y_{n+1}\right)$ is given by

$$
\varphi(x, y)=e^{-n}\left[\left(y-y_{n}\right)\left(-\sum_{n+1}^{\infty} \psi_{k}(x)\right)-\left(y-y_{n+1}\right)\left(-\sum_{n}^{\infty} \psi_{k}(x)\right)\right] .
$$


This $\varphi$ is actually defined only for $x \in \mathbf{R}$ and $y \geq y_{1}$. To extend it to the case $y<y_{1}$ we define $\varphi$ for $x \in \mathbf{R}$ and $y_{1}-y_{n+1}<y<y_{1}-y_{n}$ as follows:

$$
\varphi(x, y)=\frac{1}{y_{n+1}-y_{n}}\left[\left(y-y_{1}+y_{n}\right) f_{n+1}(x)-\left(y-y_{1}+y_{n+1}\right) f_{n}(x)\right] .
$$

This $\varphi$ is in $M^{2}$ for sure. Now we need to show that $\varphi$ is not in $L^{\infty}\left(\mathbf{R}^{2}\right)$. Let $x>0$ and $x \leq e^{-k}$. Then $\ln x \leq-k$ or $\ln \frac{1}{x} \geq k$. So

$$
-f_{n}(x)=x \sum_{n}^{\left\lfloor\ln \frac{1}{x}\right\rfloor} \frac{e^{k}}{k}-\sum_{n}^{\left\lfloor\ln \frac{1}{x}\right\rfloor} \frac{1}{k} \geq \sum_{n}^{\left\lfloor\ln \frac{1}{x}\right\rfloor} \frac{1}{k} .
$$

Now as $x \rightarrow 0$, then $\left\lfloor\ln \frac{1}{x}\right\rfloor \rightarrow \infty$. Hence $\ln \left\lfloor\ln \frac{1}{x}\right\rfloor \rightarrow \infty$. Thus $f_{n}(x) \rightarrow \infty$, which implies that $\varphi(x, y) \rightarrow \infty$. So $\varphi \notin L^{\infty}\left(\mathbf{R}^{2}\right)$.

Remark 3. This counterexample found is a $B M O$ function by Theorem 2 .

One can prove Proposition 2 by using Fourier transforms, but it is only an existence proof. One may also wonder under what additional conditions $M^{2}$ will be in $L^{\infty}\left(\mathbf{R}^{\mathbf{2}}\right)$. The answer is given by

Proposition 3. Let $\varphi \in L^{2}\left(\mathbf{R}^{\mathbf{2}}\right)$ be such that $\frac{\partial \varphi}{\partial x}, \frac{\partial \varphi}{\partial y}$ and $\frac{\partial^{2} \varphi}{\partial x \partial y}$ are all in $L^{2}\left(\mathbf{R}^{\mathbf{2}}\right)$. Then $\hat{\varphi} \in L^{1}\left(\mathbf{R}^{\mathbf{2}}\right)$ and hence $\varphi \in L^{\infty}\left(\mathbf{R}^{\mathbf{2}}\right)$.

\section{Classes of Self-adjoint $\square+V$ ON $L^{2}\left(\mathbf{R}^{\mathbf{n}}\right), n \geq 3$}

Remark 4. We will state theorems and propositions in the $n$-dimensional case, but we will only prove them for $n=3$ since the proofs are the same for $n>3$.

Let us first discuss the following Cauchy problem:

$$
\left\{\begin{array}{l}
u_{t t}-u_{x x}-u_{y y}=f(x, y, t), \\
u(x, y, 0)=\varphi(x, y) ; u_{t}(x, y, 0)=\psi(x, y) .
\end{array} \quad(x, y, t) \in \mathbf{R}^{2} \times \mathbf{R}^{+},\right.
$$

Now let us take the Fourier transform of $(I)$ in the $(x, y)$-plane only. We get

$$
\left\{\begin{array}{l}
\hat{u}_{t t}+\left(\eta^{2}+\xi^{2}\right) \hat{u}=\hat{f}(\eta, \xi, t) \\
\hat{u}(\eta, \xi, 0)=\hat{\varphi}(\eta, \xi) ; \hat{u}_{t}(\eta, \xi, 0)=\hat{\psi}(\eta, \xi) .
\end{array}\right.
$$

$(\hat{I})$ is a second-order ODE in $t$ that has the following solution:

$$
\begin{aligned}
\hat{u}_{\eta, \xi}(t)=\hat{\varphi}(\eta, \xi) \cos ( & \left.\sqrt{\eta^{2}+\xi^{2}}\right)+\frac{\hat{\psi}(\eta, \xi)}{\sqrt{\eta^{2}+\xi^{2}}} \sin \left(t \sqrt{\eta^{2}+\xi^{2}}\right) \\
& +\int_{0}^{t} \hat{f}(\eta, \xi, s) \frac{\sin \sqrt{\eta^{2}+\xi^{2}}(t-s)}{\sqrt{\eta^{2}+\xi^{2}}} d s .
\end{aligned}
$$

The previous holds for $t \geq 0$. For $t<0,(I)$ becomes, after setting $\tilde{u}(x, y, t)=$ $u(x, y,-t)$,

$(\tilde{I}) \quad\left\{\begin{array}{l}\tilde{u}_{t t}-\tilde{u}_{x x}-\tilde{u}_{y y}=f(x, y,-t), \\ \tilde{u}(x, y, 0)=\varphi(x, y) ; \tilde{u}_{t}(x, y, 0)=-\psi(x, y) .\end{array} \quad(x, y, t) \in \mathbf{R}^{2} \times \mathbf{R}^{-}\right.$,

Now we "fourier" everything in the $(x, y)$-plane to obtain

$$
\left\{\begin{array}{l}
\hat{\tilde{u}}_{t t}+\left(\eta^{2}+\xi^{2}\right) \hat{\tilde{u}}=\hat{f}(\eta, \xi,-t), \\
\hat{\tilde{u}}(\eta, \xi, 0)=\hat{\varphi}(\eta, \xi) ; \hat{\tilde{u}}_{t}(\eta, \xi, 0)=-\hat{\psi}(\eta, \xi),
\end{array}\right.
$$


which has the following solution:

$$
\begin{array}{r}
\hat{u}_{\eta, \xi}(-t)=\hat{\tilde{u}}_{\eta, \xi}(t)=\hat{\varphi}(\eta, \xi) \cos \left(t \sqrt{\eta^{2}+\xi^{2}}\right)-\frac{\hat{\psi}(\eta, \xi)}{\sqrt{\eta^{2}+\xi^{2}}} \sin \left(t \sqrt{\eta^{2}+\xi^{2}}\right) \\
+\int_{0}^{-t} \hat{f}(\eta, \xi, s) \frac{\sin \sqrt{\eta^{2}+\xi^{2}}(-t-s)}{\sqrt{\eta^{2}+\xi^{2}}} d s,
\end{array}
$$

and this holds for $t<0$. After adding up (2) and (3), one gets for $t \in \mathbf{R}$ :

$$
\begin{array}{r}
\hat{u}_{\eta, \xi}(t)=2 \hat{\varphi}(\eta, \xi) \cos \left(t \sqrt{\eta^{2}+\xi^{2}}\right)+\int_{0}^{t} \hat{f}(\eta, \xi, s) \frac{\sin \sqrt{\eta^{2}+\xi^{2}}(t-s)}{\sqrt{\eta^{2}+\xi^{2}}} d s \\
+\int_{0}^{-t} \hat{f}(\eta, \xi, s) \frac{\sin \sqrt{\eta^{2}+\xi^{2}}(-t-s)}{\sqrt{\eta^{2}+\xi^{2}}} d s-\hat{u}_{\eta, \xi}(-t) .
\end{array}
$$

Now we can change our Cauchy problem by introducing different initial conditions mainly for the $t$ variable; i.e., instead of working on the intervals $(0, t)$ and $(-t, 0)$, we will be working on $\left(\frac{t+\alpha}{2}, t\right)$ and $\left(\alpha, \frac{t+\alpha}{2}\right)$ where $\alpha$ is to be chosen freely. Now given $u \in C_{0}^{\infty}$, then we can regard it as a solution of the Cauchy problem with $u \in C_{0}^{\infty}\left(\mathbf{R}^{3}\right)$ where $f=\square u, u(x, y, 0)=\varphi(x, y)$ and $u_{t}(x, y, 0)=\psi(x, y)$. Then (4) will be:

$$
\begin{aligned}
& \hat{u}(\eta, \xi, t)=2 \hat{u}\left(\eta, \xi, \frac{t+\alpha}{2}\right) \cos \left[\sqrt{\eta^{2}+\xi^{2}}(t)\right]-\hat{u}(\eta, \xi, \alpha) \\
& +\int_{\frac{t+\alpha}{2}}^{t} \hat{f}(\eta, \xi, s) \frac{\sin \sqrt{\eta^{2}+\xi^{2}}(t-s)}{\sqrt{\eta^{2}+\xi^{2}}} d s+\int_{\frac{t+\alpha}{2}}^{\alpha} \hat{f}(\eta, \xi, s) \frac{\sin \sqrt{\eta^{2}+\xi^{2}}(\alpha-s)}{\sqrt{\eta^{2}+\xi^{2}}} d s .
\end{aligned}
$$

Proposition 4. Let $n \geq 1$. Let $u \in M^{n+1}$. Then $\|u(\ldots, t)\|_{L^{2}\left(\mathbf{R}^{\mathbf{n}}\right)} \in L^{\infty}(\mathbf{R})$ and we have

$$
\sup _{t \in \mathbf{R}}\|u(\ldots, t)\|_{L^{2}\left(\mathbf{R}^{\mathbf{n}}\right)} \leq a\|\square u\|_{L^{2}\left(\mathbf{R}^{\mathbf{n}+1}\right)}+b\|u\|_{L^{2}\left(\mathbf{R}^{\mathbf{n}+1}\right)} .
$$

Proof. We shall prove this proposition for functions in $C_{0}^{\infty}\left(\mathbf{R}^{3}\right)$ first 1 Then the result follows for functions in $M^{3}$ since $C_{0}^{\infty}\left(\mathbf{R}^{3}\right)$ is dense in $M^{3}$ in the graph norm of $\square$.

We choose $\alpha$ such that $|t-\alpha| \leq 1$, and then we obtain:

$|\hat{u}(\eta, \xi, t)| \leq 2\left|\hat{u}\left(\eta, \xi, \frac{t+\alpha}{2}\right)\right|+\tilde{c} \int_{\frac{t+\alpha}{2}}^{t}|\hat{f}(\eta, \xi, s)| d s+\tilde{c} \int_{\frac{t+\alpha}{2}}^{\alpha}|\hat{f}(\eta, \xi, s)| d s+|\hat{u}(\eta, \xi, \alpha)|$,

where we have used the fact that $\left|\frac{\sin X}{X}\right| \leq 1$. Using the Cauchy-Schwarz inequality and taking the squares of both sides, we will have

$$
\begin{array}{r}
|\hat{u}(\eta, \xi, t)|^{2} \leq \tilde{c}\left|\hat{u}\left(\eta, \xi, \frac{t+\alpha}{2}\right)\right|^{2}+\tilde{c} \int_{\frac{t+\alpha}{2}}^{t}|\hat{f}(\eta, \xi, s)|^{2} d s+\tilde{c} \int_{\frac{t+\alpha}{2}}^{\alpha}|\hat{f}(\eta, \xi, s)|^{2} d s \\
+\tilde{c}|\hat{u}(\eta, \xi, \alpha)|^{2} .
\end{array}
$$

\footnotetext{
${ }^{1} C_{0}^{\infty}\left(\mathbf{R}^{\mathbf{3}}\right)$ is the space of infinitely differentiable functions with compact support.
} 
Then integrate (6) with respect to $\eta$ and $\xi$ in $\mathbf{R}^{\mathbf{2}}$, and bound the integral of $|\hat{f}|^{2}$ by the one over $\mathbf{R}^{\mathbf{3}}$ to obtain

$$
\begin{aligned}
\iint_{\mathbf{R}^{2}}|\hat{u}(\eta, \xi, t)|^{2} d \eta d \xi \leq \tilde{c} \iint_{\mathbf{R}^{2}}\left|\hat{u}\left(\eta, \xi, \frac{t+\alpha}{2}\right)\right|^{2} d \eta d \xi+\tilde{c} \iiint_{\mathbf{R}^{3}}|\hat{f}(\eta, \xi, s)|^{2} d \eta d \xi d s \\
+\tilde{c} \iiint_{\mathbf{R}^{3}}|\hat{f}(\eta, \xi, s)|^{2} d \eta d \xi d s+\tilde{c} \iint_{\mathbf{R}^{2}}|\hat{u}(\eta, \xi, \alpha)|^{2} d \eta d \xi
\end{aligned}
$$

Now integrate everything w.r.t. $\alpha$ in the segment $|t-\alpha| \leq 1$ and bound again by integrals over $\mathbf{R}^{3}$ to get

$$
\begin{aligned}
\iint_{\mathbf{R}^{2}}|\hat{u}(\eta, \xi, t)|^{2} d \eta d \xi \leq \tilde{c} & \iiint_{\mathbf{R}^{3}}\left|\hat{u}\left(\eta, \xi, \frac{t+\alpha}{2}\right)\right|^{2} d \eta d \xi d \alpha+\tilde{c} \iiint_{\mathbf{R}^{3}}|\hat{f}(\eta, \xi, s)|^{2} d \eta d \xi d s \\
& +\tilde{c} \iiint_{\mathbf{R}^{3}}|\hat{f}(\eta, \xi, s)|^{2} d \eta d \xi d s+\tilde{c} \iiint_{\mathbf{R}^{\mathbf{3}}}|\hat{u}(\eta, \xi, \alpha)|^{2} d \eta d \xi d \alpha .
\end{aligned}
$$

Thus by taking the supremum in $t$ over $\mathbf{R}$ and by using the Plancherel theorem, one has

$$
\sup _{t \in \mathbf{R}}\|u(., ., t)\|_{L^{2}\left(\mathbf{R}^{2}\right)} \leq \tilde{c}\|\square u\|_{L^{2}\left(\mathbf{R}^{3}\right)}+\tilde{c}\|u\|_{L^{2}\left(\mathbf{R}^{3}\right)} .
$$

The estimate for functions in $M^{3}$ then follows by the denseness of $C_{0}^{\infty}$ in $M^{3}$.

Theorem 4. Let $\square$ be the wave operator defined on $L^{2}\left(\mathbf{R}^{\mathbf{n}+\mathbf{1}}\right)$. Let $V$ be a realvalued function such that $\int_{-\infty}^{\infty}\|V(\ldots, t)\|_{L^{\infty}\left(\mathbf{R}^{\mathbf{n}}\right)}^{2} d t<\infty$. Then $\square+V$ is self-adjoint on $\mathcal{D}(\square)$.

Proof. The proof is very similar to the one of Theorem 3.

Proposition 5. Let $V=V_{1}+V_{2}$ be real-valued such that $V_{1}$ is as in Theorem 4 and $V_{2} \in L^{\infty}\left(\mathbf{R}^{\mathbf{n}+\mathbf{1}}\right)$. Then $\square+V$ is self-adjoint on $\mathcal{D}(\square)$.

By going back to equation (4), we can have a better estimate than the one in Proposition 4 and we have

Proposition 6. Let $n \geq 2$, and let $u \in M^{n+1}$. Then $\|u(\ldots, t)\|_{L^{r}\left(\mathbf{R}^{\mathbf{n}}\right)} \in L^{\infty}(\mathbf{R})$, $2<r<\frac{2 n}{n-1}$ and we have

$$
\sup _{t \in \mathbf{R}}\|u(., ., t)\|_{L^{r}\left(\mathbf{R}^{\mathbf{n}}\right)} \leq a\|\square u\|_{L^{2}\left(\mathbf{R}^{\mathbf{n}+\mathbf{1}}\right)}+b\|u\|_{L^{2}\left(\mathbf{R}^{\mathbf{n}+\mathbf{1}}\right)} .
$$

In order to do that we will need some lemmas.

Lemma 3 (Sobolev's inequality). Let $n \geq 2$. Let $f \in H^{1}\left(\mathbf{R}^{\mathbf{n}}\right)$. Then $f \in L^{q}\left(\mathbf{R}^{\mathbf{n}}\right)$ for $2 \leq q<\frac{2 n}{n-2}$ (with $n=2$ giving $q=\infty$ ) and we have

$$
\|f\|_{q} \leq a\|\nabla f\|_{2}+b\|f\|_{2} .
$$

We recall the following interpolation result:

Proposition 7. Let $u(t) \in L^{2}\left(\mathbf{R}^{\mathbf{n}}\right)$ be such that also $u(t) \in L_{w}^{p}\left(\mathbf{R}^{\mathbf{n}}\right)$ Then $u(t) \in L^{r}\left(\mathbf{R}^{\mathbf{n}}\right)$ for $2<r<p$.

${ }^{2} H^{1}\left(\mathbf{R}^{\mathbf{n}}\right)$ is the Sobolev space $\left\{f \in L^{2}\left(\mathbf{R}^{\mathbf{n}}\right): \nabla f \in L^{2}\left(\mathbf{R}^{\mathbf{n}}\right\}\right.$.

${ }^{3}$ The $L^{p}$-weak norm of $u$ will be denoted by $\|\cdot\|_{p, w}$. 
Lemma 4. Assume for all $\epsilon>0$, there exist $V, g$ such that $u(t)=V+g,\|V\|_{2} \leq d \epsilon$ and $\|g\|_{q} \leq \frac{c}{\epsilon}$ where $c, d$ are two constants. Then $u(t) \in L_{w}^{p}\left(\mathbf{R}^{\mathbf{n}}\right)$ where $p=\frac{4 q}{2+q}$ and $2 \leq q<\frac{2 n}{n-2}$.

Proof. We only prove the lemma for $n=2$. Let $E_{\lambda}=\{x:|u(x)| \geq \lambda\}$. Then, since $u=V+g$,

so that

$$
E_{\lambda} \subset\left\{x:|V(x)| \geq \frac{\lambda}{2}\right\} \cup\left\{x:|g(x)| \geq \frac{\lambda}{2}\right\}
$$

$$
\left|E_{\lambda}\right| \subset\left|\left\{x:|V(x)| \geq \frac{\lambda}{2}\right\}\right|+\left|\left\{x:|g(x)| \geq \frac{\lambda}{2}\right\}\right| .
$$

Then by using the Chebyshev inequality, we get $\left|E_{\lambda}\right| \leq 4 \lambda^{-2}\|V\|_{2}^{2}+2^{q} \lambda^{-q}\|g\|_{q}^{q}$. But we have the freedom to choose any $\epsilon(\lambda)>0$. So take $\epsilon(\lambda)=\lambda^{b}$ (b to be determined). With this choice we obtain

$$
\left|E_{\lambda}\right| \leq 4 d^{2} \lambda^{2 b-2}+2^{q} c^{q} \lambda^{-b q-q} \text { or } \lambda^{p}\left|E_{\lambda}\right| \leq 4 d^{2} \lambda^{p+2 b-2}+2^{q} c^{q} \lambda^{p-b q-q} .
$$

Then $u(t)$ belongs to $L_{w}^{p}\left(\mathbf{R}^{2}\right)$ if and only if $\sup _{\lambda>0}\left[4 d^{2} \lambda^{p+2 b-2}+2^{q} c^{q} \lambda^{p-b q-q}\right]<\infty$, which is only possible if $b=\frac{2-p}{2}$ and $p=\frac{4 q}{2+q}$.

Now we prove Proposition 6.

Proof. Let $u \in C_{0}^{\infty}$. We use (5) to show that $u(t)$ is in $L^{r}\left(\mathbf{R}^{\mathbf{2}}\right), 2 \leq r<4(n=2$ gives $q<\infty$ and hence $r<4)$. We set $u(t)=V+g$. So $\hat{u}(t)=\hat{V}+\hat{g}$,

$$
\hat{V}(\eta, \xi)=2 \hat{u}\left(\eta, \xi, \frac{t+\alpha}{2}\right) \cos \left[\sqrt{\eta^{2}+\xi^{2}}(t)\right]-\hat{u}(\eta, \xi, \alpha)
$$

and

$$
\begin{aligned}
\hat{g}(\eta, \xi)=\int_{\frac{t+\alpha}{2}}^{t} \hat{f}(\eta, \xi, s) & \frac{\sin \sqrt{\eta^{2}+\xi^{2}}(t-s)}{\sqrt{\eta^{2}+\xi^{2}}} d s \\
& +\int_{\frac{t+\alpha}{2}}^{t} \hat{f}(\eta, \xi, s) \frac{\sin \sqrt{\eta^{2}+\xi^{2}}(\alpha-s)}{\sqrt{\eta^{2}+\xi^{2}}} d s
\end{aligned}
$$

where $\hat{f} \in L^{2}\left(\mathbf{R}^{\mathbf{3}}\right)$.

We observe that both $\hat{V}$ and $\hat{g}$ depend on $\alpha$. We will first show that $\nabla g \in L^{2}\left(\mathbf{R}^{\mathbf{2}}\right)$ so that $g \in L^{q}\left(\mathbf{R}^{2}\right), 2 \leq q<\infty$, by Lemma 3 since $g \in L^{2}\left(\mathbf{R}^{\mathbf{2}}\right)$. Then we show that $V \in L^{2}\left(\mathbf{R}^{2}\right)$. We only do the cases $\hat{u}\left(\eta, \xi, \frac{t+\alpha}{2}\right) \cos \left[\sqrt{\eta^{2}+\xi^{2}}(t)\right]$ and $\int_{\frac{t+\alpha}{2}}^{t} \hat{f}(\eta, \xi, s) \frac{\sin \sqrt{\eta^{2}+\xi^{2}}(t-s)}{\sqrt{\eta^{2}+\xi^{2}}} d s$, which we also denote by $\hat{V}(\eta, \xi)$ and $\hat{g}(\eta, \xi)$. The proof of the other two cases is simply the same.

Also, the assumptions we made in Lemma 4 suggested that $\|V\|_{2} \leq d \epsilon$ and $\|g\|_{q} \leq \frac{c}{\epsilon}$. So here we find the constants $c$ and $d$ explicitly and, with no surprise, they depend on $\|u\|_{L^{2}\left(\mathbf{R}^{3}\right)}$ and $\|\square u\|_{L^{2}\left(\mathbf{R}^{3}\right)}$.

We also bear in mind that we still have the freedom to choose $\alpha$. We also give details of why we have $\nabla g \in L^{2}\left(\mathbf{R}^{\mathbf{2}}\right)$. We have $\hat{u}=\hat{V}+\hat{g}$ where

$$
\hat{V}(\eta, \xi)=2 \hat{u}\left(\eta, \xi, \frac{t+\alpha}{2}\right) \cos \left[\sqrt{\eta^{2}+\xi^{2}}(t)\right]
$$

and

$$
\hat{g}(\eta, \xi)=\int_{\frac{t+\alpha}{2}}^{t} \hat{f}(\eta, \xi, s) \frac{\sin \sqrt{\eta^{2}+\xi^{2}}(t-s)}{\sqrt{\eta^{2}+\xi^{2}}} d s \text { where } \hat{f} \in L^{2}\left(\mathbf{R}^{\mathbf{3}}\right) .
$$


Also, $\hat{g} \in L^{2}\left(\mathbf{R}^{\mathbf{2}}\right)$ and since

$$
-i \eta \frac{\sin \sqrt{\eta^{2}+\xi^{2}}(t-s)}{\sqrt{\eta^{2}+\xi^{2}}},-i \xi \frac{\sin \sqrt{\eta^{2}+\xi^{2}}(t-s)}{\sqrt{\eta^{2}+\xi^{2}}} \in L^{\infty}\left(\mathbf{R}^{\mathbf{2}}\right),
$$

then $-i \eta \hat{g},-i \xi \hat{g} \in L^{2}\left(\mathbf{R}^{\mathbf{2}}\right)$, i.e., $\widehat{\nabla g} \in L^{2}\left(\mathbf{R}^{\mathbf{2}}\right)$. So by Sobolev's inequality we have $g \in L^{q}\left(\mathbf{R}^{\mathbf{2}}\right), 2 \leq q<\infty$. We also have

$$
\|\nabla g\|_{L^{2}\left(\mathbf{R}^{2}\right)}^{2}=\|\widehat{\nabla g}\|_{L^{2}\left(\mathbf{R}^{2}\right)}^{2}=\iint_{\mathbf{R}^{2}}|\eta \hat{g}(\eta, \xi)|^{2} d \eta d \xi+\iint_{\mathbf{R}^{2}}|\xi \hat{g}(\eta, \xi)|^{2} d \eta d \xi .
$$

But

and

$$
\eta^{2}|\hat{g}(\eta, \xi)|^{2} \leq a^{2}|t-\alpha| \int_{\frac{t+\alpha}{2}}^{t}|\hat{f}(\eta, \xi, s)|^{2} d s
$$

$$
\xi^{2}|\hat{g}(\eta, \xi)|^{2} \leq a^{2}|t-\alpha| \int_{\frac{t+\alpha}{2}}^{t}|\hat{f}(\eta, \xi, s)|^{2} d s
$$

So by choosing $\alpha$ such that $|t-\alpha| \leq \frac{1}{\epsilon^{2}}$ we get

$$
\|\nabla g\|_{L^{2}\left(\mathbf{R}^{2}\right)}^{2} \leq \frac{2 a^{2}}{\epsilon^{2}} \iint_{\mathbf{R}^{2}} \int_{\frac{t+\alpha}{2}}^{t}|\hat{f}(\eta, \xi, s)|^{2} d \eta d \xi d s .
$$

Thus

$$
\|\nabla g\|_{L^{2}\left(\mathbf{R}^{2}\right)} \leq \frac{c}{\epsilon}\|\square u\|_{L^{2}\left(\mathbf{R}^{3}\right)} .
$$

In a similar way, one gets $\|g\|_{L^{2}\left(\mathbf{R}^{2}\right)} \leq \frac{c}{\epsilon^{\frac{3}{2}}}\|\square u\|_{L^{2}\left(\mathbf{R}^{3}\right)}$. So for $\epsilon \geq 1$ we have $\|g\|_{L^{2}\left(\mathbf{R}^{2}\right)} \leq \frac{c}{\epsilon}\|\square u\|_{L^{2}\left(\mathbf{R}^{3}\right)}$. Also, for $\epsilon \leq 1$ one has to use $\hat{g}=\hat{u}-\hat{V}$ and the estimate we will get for $\|V\|_{L^{2}\left(\mathbf{R}^{2}\right)}$ below and the fact that $u$ belongs to $L^{2}\left(\mathbf{R}^{3}\right)$.

For $V$ we have

$$
\hat{V}(\eta, \xi)=2 \hat{u}\left(\eta, \xi, \frac{t+\alpha}{2}\right) \cos \left[\sqrt{\eta^{2}+\xi^{2}}(t)\right]
$$

Then

$$
\|\hat{V}\|_{L^{2}\left(\mathbf{R}^{2}\right)}^{2} \leq 4 \iint_{\mathbf{R}^{\mathbf{2}}}\left|\hat{u}\left(\eta, \xi, \frac{t+\alpha}{2}\right)\right|^{2} d \eta d \xi,
$$

and then by integrating w.r.t. $\alpha \in\left\{\alpha:|t-\alpha| \leq \frac{1}{\epsilon^{2}}\right\}$ one obtains

$$
\int_{t-\frac{1}{\epsilon^{2}}}^{t+\frac{1}{\epsilon^{2}}}\|\hat{V}\|_{L^{2}\left(\mathbf{R}^{2}\right)}^{2} d \alpha \leq 4 \iint_{\mathbf{R}^{2}} \int_{t-\frac{1}{\epsilon^{2}}}^{t+\frac{1}{\epsilon^{2}}}\left|\hat{u}\left(\eta, \xi, \frac{t+\alpha}{2}\right)\right|^{2} d \eta d \xi d \alpha \leq 4\|\hat{u}\|_{L^{2}\left(\mathbf{R}^{3}\right)}^{2} .
$$

Then there exists $\alpha \in\left(t-\frac{1}{\epsilon^{2}}, t+\frac{1}{\epsilon^{2}}\right)$ s.t. $\|\hat{V}\|_{L^{2}\left(\mathbf{R}^{2}\right)}^{2} \leq \frac{4\|\hat{u}\|_{L^{2}\left(\mathbf{R}^{3}\right)}^{2}}{\frac{2^{2}}{\epsilon^{2}}}$. So $\|V\|_{L^{2}\left(\mathbf{R}^{2}\right)} \leq$ $d \epsilon\|u\|_{L^{2}\left(\mathbf{R}^{3}\right)}$. So one has

$$
\sup _{\lambda>0}\left[4 d^{2} \lambda^{p-2+2 b}+2^{q} c^{q} \lambda^{p-q-b q}\right]=\tilde{c}\|u\|_{L^{2}\left(\mathbf{R}^{3}\right)}^{2}+\tilde{c}\|\square u\|_{L^{2}\left(\mathbf{R}^{3}\right)}^{q} .
$$

We have by Proposition 7 that $\|u(t)\|_{L^{r}\left(\mathbf{R}^{2}\right)} \leq \tilde{c}\|u(t)\|_{2}+\tilde{c}\|u(t)\|_{p, w}$. So by using (8), Proposition 4, scaling by a suitable constant and taking the supremum in $t$ over $\mathbf{R}$, one obtains

$$
\sup _{t \in \mathbf{R}}\|u(., ., t)\|_{L^{r}\left(\mathbf{R}^{2}\right)} \leq a\|\square u\|_{L^{2}\left(\mathbf{R}^{3}\right)}+b\|u\|_{L^{2}\left(\mathbf{R}^{3}\right)} .
$$


Remark 5. The constant $a$ in (9) may be made as small as we would like.

Theorem 5. Let $n \geq 2$. Let $\square$ be the wave operator defined on $L^{2}\left(\mathbf{R}^{\mathbf{n}+\mathbf{1}}\right)$. Let $V$ be a real-valued function such that $\int_{-\infty}^{\infty}\|V(\ldots, t)\|_{L^{s}\left(\mathbf{R}^{\mathbf{n}}\right)}^{2} d t<\infty$ for $\frac{1}{s}=\frac{1}{2}-\frac{1}{r}$ and $2 \leq r<\frac{2 n}{n-1}$. Then $\square+V$ is self-adjoint on $\mathcal{D}(\square)$.

Proof. Again we only prove the theorem for $n=2$. We have by Hölder's inequality, for $\frac{1}{2}=\frac{1}{r}+\frac{1}{s}, 2 \leq r<4$ :

$$
\begin{array}{rl}
\iint_{\mathbf{R}^{2}}|V(x, y, t) u(x, y, t)|^{2} & d x d y \leq\|V(., ., t)\|_{L^{s}\left(\mathbf{R}^{2}\right)}^{2} \cdot\|u(., ., t)\|_{L^{r}\left(\mathbf{R}^{2}\right)}^{2} \\
\leq \sup _{t \in \mathbf{R}}\|u(., ., t)\|_{L^{r}\left(\mathbf{R}^{2}\right)}^{2}\left(\|V(., ., t)\|_{L^{s}\left(\mathbf{R}^{2}\right)}^{2}\right) .
\end{array}
$$

Then by using Proposition 6 and by integrating w.r.t. $t$ over $\mathbf{R}$ one gets

$$
\|V u\|_{2}^{2} \leq a\left(\int_{\mathbf{R}}\|V(., ., t)\|_{L^{s}\left(\mathbf{R}^{2}\right)}^{2} d t\right)\|\square u\|_{2}^{2}+b\left(\int_{\mathbf{R}}\|V(., ., t)\|_{L^{s}\left(\mathbf{R}^{2}\right)}^{2} d t\right)\|u\|_{2}^{2} .
$$

Since we can make $a$ small enough to have $a \int_{\mathbf{R}}\|V(., ., t)\|_{L^{\infty}\left(\mathbf{R}^{2}\right)}^{2} d t<1$ we conclude by the Kato-Rellich perturbation theorem that $\square+V$ is self-adjoint on $\mathcal{D}(\square)$.

\section{Open PROBLEMS}

This paper contains results from my Ph.D. thesis. I have a series of questions that I would like to know the answers to:

1) If $V$ is real-valued and $V \in L^{2}\left(\mathbf{R}^{\mathbf{n}}\right)$, then will $\square+V$ be self-adjoint?

2) If $V \geq 0$ is real-valued and $V \in L_{l o c}^{2}\left(\mathbf{R}^{\mathbf{n}}\right)$, then will $\square+V$ be self-adjoint? (We insist on $V$ being positive since if it has no sign.) Then there are examples that show that this may fail, and they exploit the non-self-adjointness of the one-dimensional Hamiltonian for some classes of potentials [3].

3) In Section 4, can we have larger spaces than the ones found in Section 2 and 4 and which include $M^{n}, n \geq 3$ ?

\section{ACKNOWLEDGMENT}

I should be very grateful to my supervisor Prof. Alexander M. Davie for the helpful suggestions, comments and hints. I also would like to thank the Algerian Government for the full scholarship it has provided me with.

\section{REFERENCES}

1. E. H. Lieb, M. Loss, Analysis, Vol. 14, Graduate Studies in Mathematics, American Mathematical Society, 2001 (2nd edition). MR 2001i:00001

2. M. Reed, B. Simon, Methods of Modern Mathematical Physics, Vol.1, Functional Analysis, Academic Press, 1972. MR 58:12429a

3. M. Reed, B. Simon, Methods of Modern Mathematical Physics, Vol.2, Fourier Analysis, Selfadjointness, Academic Press, 1975. MR 58:12429b

4. J. Duoandikoetxea, Fourier Analysis, Graduate Studies in Mathematics Vol. 29, American Mathematical Society, 2001. MR 2001k:42001

School of Mathematics, University of Edinburgh, JCMB, Mayfield Road, Edinburgh, EH9 3JZ, UNiTED KingDOM

E-mail address: mortad@maths.ed.ac.uk

E-mail address: hichem1978@yahoo.fr 